\title{
MAXILLOFACIAL PATHOLOGY DETECTION USING AN EXTENDED A CONTRARIO APPROACH COMBINED WITH FUZZY LOGIC
}

\author{
Nedra Nefzi and Nawrès Khlifa \\ j.nedra@gmail.com, khalifa_nawres@yahoo.com \\ National Engineering School of Tunis \\ University of Tunis ElManar-Tunisia
}

\begin{abstract}
In this work, we develop a method for pathology detection of the maxillary-facial in 3D CT medical images. Our method is based on "a contrario" approach that is a statistical region based method. To do this, we detect significant changes in regions previously delineated by a physician anatomist. We apply a decision algorithm based on fuzzy logic on the changes detected and we get a decision with a degree of uncertainty. Tests conducted on a basis of real images have shown the performance and robustness of the proposed approach.
\end{abstract}

\section{KEYWORDS}

Change detection, "a contrario approach”, Fisher law, Fuzzy logic.

\section{INTRODUCTION}

With recent technological advances and the abundance of images of all kinds, the imaging world knows now a phenomenal growth that led to the diversification and multiplication of research on this theme. Among the various imaging applications, we can mention that on the medical world.

The tomographic images based on the X-rays, the MRI images, the ultrasound images or the optical images are various types of medical images. These last ones bubble with data and it would be wise to be able to get the maximum of useful information. Indeed, the role of treatments applied to the medical images is to generate a representation which would facilitate the interpretation of these images at first sight complicated.

In this work, we will focus particularly in the detection of craniofacial malformations, a discipline whose interest is growing day by day but whose limits are significant as the unintelligible image interpretation, decision-making and automate processing.

The malformations detection can be considered as a changes detection task. The problem is often posing in medical imaging. In the literature several methods exist and each one presents advantages and inconveniences. Notice these methods can either be used to compare two or more groups or to compare a subject with a group. We consider hereafter only the case of single-subject vs group analysis.

The essential objective of this work is to develop a method which allows changes detection in 3D images of the maxillofacial massif. We are particularly going to be interested in "a contrario" method which consists in rejecting a null hypothesis, which supposes that all the subjects are

DOI : $10.5121 /$ sipij.2011.2308 
Signal \& Image Processing : An International Journal (SIPIJ) Vol.2, No.3, September 2011

healthy. The approach, being global and effective, gives interesting results. However, this last one presents major limitations: it depends on method parameters and on the combination of the pvalues to generate the definitive decision that does not take advantage of all the values. This can alter the results.

In this paper, we suggest changing the Gaussian test, often used for approximating the distribution of the measures, by the Fisher test, the law which models at best the distribution of the measures in the presence of a limited number of samples. So, we shall try to change the combination of the p-values by a method completely automatic which considers all the values.

This paper will be organized as follows: in the second section we are going to present the state of the art as well as the method proposed, in the third section we are going to describe the extension we bring. Then in the fourth section we are going to present the results obtained. And to finish, the conclusion and the perspectives are brought in the fifth section.

\section{STATE OF ART:}

The a contrario approach that we are going to use to detect pathological zones of maxillo facial $3 \mathrm{D}$ images is based on the detection of change or variation. Before presenting our method we are briefly going to present the other methods of detection of change as the morphometry and the principal component analysis.

Approaches can be classified into two categories: On the one hand, the univariate methods consider that all voxels are mutually independent. The model parameter estimation and the subject vs group comparison are also performed voxel by voxel. One well-known example of univariate model is the General Linear Model (GLM) which is for example used in Voxel-Based Morphometry (VBM)[5]. In this case, the observation at each voxel is expressed as the output of a GLM. The success of this approach is explained by the richness of this model (covariables such as age are used as regressors in the model) and by the associated theoretical frameworks which enable to solve inference problems and to estimate relevant statistical map thresholding.

On the other hand, the multivariate methods take into account the correlation which may exist between voxels. There is no more one model per voxel but rather a model for the whole image. The purpose of these methods is to perform the statistical analysis into a low-dimensional space by reducing the space dimension of the original data (3D images in our case). The most wellknown algorithm dedicated to this problem is the principal component analysis. There also exists a great number of nonlinear dimensionality reduction techniques. Notice that more recently, methods such as the isomap [6] assume that the underlying structure of the image lies on a nonlinear manifold. When the global model has been learnt from the group, the comparison between the model and a subject can either be performed voxel by voxel (a reconstruction step is needed in this case) or directly for the whole image in the low-dimensional space.

In the following, the comparison cannot be conducted neither voxel by voxel nor for the whole image since the application is related to surgery: surgeons would like to know for a given subject the normality of specific regions they can operate. Consequently, from the information processing point of view, the statistical comparison has to be performed region by region. Considering the restricted number of available subjects in our current image database and the complexity of the facial maxillary, the use of a global approach for each region can not be envisaged (the mapping estimation between the low-dimensional space and the original image space requires indeed too many examples). Thus, we propose in this article a region-based method which does not require dimensionality reduction. The proposed approach relies on an a contrario approach [7][8] that we have adapted and extended to deal with single-subject vs group analysis. 
Signal \& Image Processing : An International Journal (SIPIJ) Vol.2, No.3, September 2011

The 'a contrario' approach recently introduced is based on the detection of the rare changes which have a low probability of occurrence [1]. In other words the more an event has a low probability to appear at random, the more it is significant [4].

By modeling the differences which can appear accidentally between the image to be tested and the images of the base, the abnormalities are going to be detected by looking for the differences which are statistically too big to be the result of the null hypothesis. Thus these differences just have to be significantly bigger than the differences observed by the fate.

The proposed method is based on the contrario approach; we begin by reminding its principle before presenting the extensions we propose.

\section{THE EXTENDED A CONTRARIO APPROACH:}

The 'a contrario' approach is based on the validity of the null hypothesis by subjecting it to various tests. It means defining thresholds from which the observed changes will be considered significant; but it is interesting not to neglect the probability of false alarms. So we can consider the method as being reliable if the hope of the number of false alarms for a given image is lower in $\varepsilon[2]$.

The 'a contrario' approach is based on the analysis of the morphological information extracted from the subjects. These last ones are represented by fields of deformations [6]. This approach allows to combine several types of information (generally combined by the operator OR).

In this approach, the 'a contrario model which we wish to reject corresponds to the null hypothesis or still Ho. The null hypothesis is rejected to the voxel V if a rare event is observed there.

Considering the number of voxels which a region can contain, the reject of this hypothesis can lead to a very important number of false alarms. To by-pass this constraint, Ho is thus rejected, for a given region, as soon as a significant number of rare events is observed there.

The approach a contrario analyzes the information extracted from every region, so allowing a decision with regard to every region and not with regard to a voxel.

The decision system is defined by an application of the space of the observations in the whole of the possible hypotheses. This approach uses a set of thresholds to determine what a rare event is. Remain to find those who best reject the null hypothesis.

Denote by $X$ a random variable associated with a measure of changes [3] and $S_{j}: j=1 \ldots N_{s}$ : a set of thresholds.

We consider the event: $\mathrm{E}_{\mathrm{s}, \mathrm{R}}=\left\{\right.$ at least $\mathrm{K}_{\mathrm{j}}$ rare events are observed in $\mathrm{R}$ for the threshold $\left.\mathrm{Sj}\right\}$.

$\mathrm{R}$ is a region compouned of $\mathrm{n}$ voxels and $\mathrm{K}_{\mathrm{j}}$ the number of rare events observed in this region for the threshold $S_{j}$.

The probability corresponding to the event $\mathrm{E}_{\mathrm{s} \mathrm{j}, \mathrm{R}}$ is the following one:

$$
\mathrm{P}\left(E_{s j, R}\right)=\sum_{K=K j}^{n} C_{j}^{k} \mathrm{P}\left(X>S_{j}\right)^{k} *\left(1-P\left(X>S_{j}\right)\right)^{n-k}
$$

Let NR the number of regions of the image, a region $\mathrm{R}$ is said $\varepsilon$-significant if and only if: 
Signal \& Image Processing : An International Journal (SIPIJ) Vol.2, No.3, September 2011

$\mathrm{N}_{\mathrm{R}} * \mathrm{~N}_{\mathrm{S}} * \min \mathrm{P}\left(\mathrm{E}_{\mathrm{sj}}, \mathrm{R}\right)<\varepsilon \quad[3]$

The quantity $\mathrm{N}_{\mathrm{R}} * \mathrm{~N}_{\mathrm{S}} *$ min $\mathrm{P}\left(\mathrm{E}_{\mathrm{sj}}, \mathrm{R}\right)<\varepsilon$ corresponds to the number of false alarms under the null hypothesis. The approach in contrario is based on 8 stages which we are going to detail.

The first phase is the preprocessing is to determinate all of the individuals on whome we will work and develop a base of healthy patients who obey to the criteria of normality and representing the diversity of the population.

The second phase is to select the null hypothesis $\mathrm{H} 0$ that consists in supposing that no difference exists between data to be compared.

The third step is to choose the statistical control of $\mathrm{H} 0$ that really depends on the nature of data and on the null hypothesis we want to control.

The fourth step is to choose the distribution of statistics which is what we get if we took all possible samples, each one drawn randomly.

The fifth phase consists in determining the reject region what consists in fixing one or two thresholds to delimit the region where we can consider that the individual does not present significant change.

The sixth phase consists in choosing the nature of the threshold, which is or one-sided or bilateral.

The seventh phase means determining the p-values which are other one than the probability to reject wrongly the a contrario model. They are beforehand determined and translate the risk of error

The last phase is the decision which is translated by the reject of a region as soon as the number of observed rare events exceeds certain threshold defined beforehand.

\section{THE PROPOSED METHOD}

Our first contribution consists to extend the a contrario approach to the comparison of two groups, one of them being normal and the other one contains the patient to be tested.

Our second contribution consists in changing the Gaussian test often used, by a Fisher test due to its robustness especially when the base of samples is reduced.

Our third contribution consists in combining p_values using the formalism of the fuzzy logic to grant to the decision obtained a certain degree of trust.

In this section, we are going to begin by reminding the principle of the Fisher test and the modification which we brought so that she can take into account two groups: the first one would be for the learning and the second to which we are going to add a patient presenting a pathology would be for the test. We present then the method we proposed to combine the p-values in a way that every result deducted of one p-value will be taken into account.

\subsection{Modeling of samples by the Fisher distribution}

We choose the Fisher law to model our database. We consider two random variables $U$ and $V$ of two independent samples, following the Fisher distribution and having respectively $n$ and $m$ as freedom degrees. 
Signal \& Image Processing : An International Journal (SIPIJ) Vol.2, No.3, September 2011

$$
5=\frac{V / n}{V / m}
$$

The approach can be divided into two stages: a stage of learning of the model «a contrario» and a stage of test of a new individual.

In the learning phase, the morphological information is extracted from an image by registration it with a reference image.

A rigid registration, at first time, is applied to correct the wrong patient positioning in the scanner. Secondly a non-rigid registration is applied to provide a deformation field representing the anatomical differences between the two images.

In other words, it is a question of estimating a transformation $\mathrm{h}$ that allows associating with every point of the reference image its correspondent in the image of the subject, so we obtain the vector field of deformation; this last one can be transformed into a measure such as the projection of the vector on three axes.

We consider $A_{i v}$ with $i=1, \ldots, n$ and $B_{j v}$ avec $j=1, \ldots, m$ variables connected to a measure $V$ respectively of the first one and the second sample containing the subject susceptible to present a pathology.

The Fisher test is the report of both variances corresponding to every sample:

$$
F=\frac{\max \left(\operatorname{Var}\left(A_{i w}\right) \operatorname{Var}\left(B_{j}\right)\right)}{\min \left(\operatorname{Var}\left(A_{i w}\right) \operatorname{Var}\left(B_{i}\right)\right)}
$$

Finally, the observation in the voxel $\mathrm{i}$ is considered as rare event for one $\mathrm{P} \_$value $\mathrm{P}$ if the quantity ( 3 ) exceeds a threshold NU appearing in the table of Fisher and concerning the numbers of the subjects in both samples.

\subsection{Combination of $\mathbf{p} \_$values by fuzzy logic}

The method which we propose is a region based method so we combined three values resultant from three p_values and this by choosing the minimum (operator OR).

This decision can be soiled by a certain degree of uncertainty, furthermore it does take into account only one p_value and ignore the others. The fuzzy logic presents a better alternative for the decision-making.[8]

The diagram 1 shows the model we have chosen as input and has a p-value $\mathrm{P}$ 


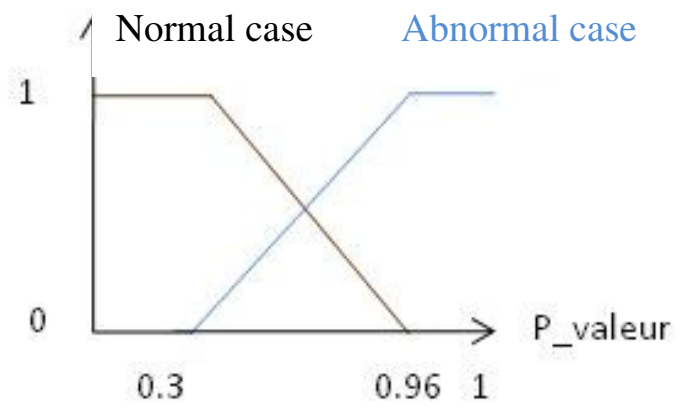

Diagram 1: graph of the chosen Model

For our fuzzy system, we chose the method of Larsen as a method of inference to combine the three p-values. For the defuzzification we have chosen the center of gravity (COG) method.

So we obtain healthy regions in $100 \%$ and the others pathological in $100 \%$. But also we are going to obtain healthy regions with a certain trust degree. What allows the doctors to investigate them.

\section{RESULTS}

The database consists of 22 subjects, 20 for the basic learning and 2 for the test database. To evaluate the method we have introduced a patient whose mandible is pathological. By applying the proposed method, we could detect pathological areas but other areas not approved by the doctor.

The test is performed with 3 measures from the projection of the deformation field on the 3 axes $\mathrm{X}, \mathrm{Y}$ and $\mathrm{Z}$. the $3 \mathrm{p}$-values are chosen empirically namely $\mathrm{P} 1=0.01, \mathrm{P} 2=0.001$ and $\mathrm{P} 3=0.005$

The result of the detection is shown in figure $1:$ we note that using a fuzzy model, the malformed regions were detected with a degree of truth of $100 \%$. But the advantage of this modeling is that it detects others regions that may represent a malformation with different degrees of truth.
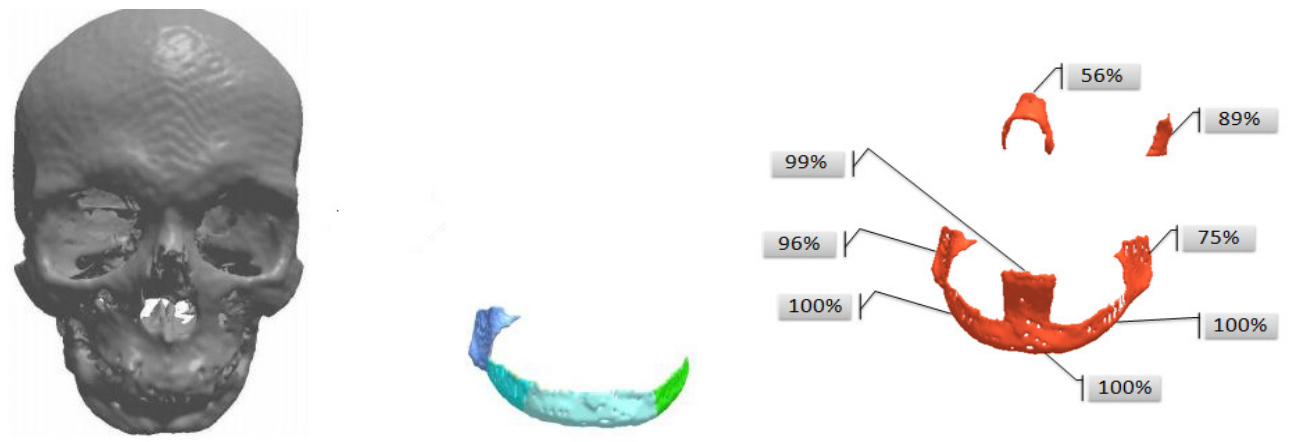

a. The deformed image b. result obtained by the a contrario approach c. result obtained by

the proposed method

Figure 1: results of abnormality detection 
Signal \& Image Processing : An International Journal (SIPIJ) Vol.2, No.3, September 2011

We can note that the result is correct and very encouraging since this detection still leaves us the benefit of the doubt for regions of slight deformation so that the doctor can explore this area more.

\section{DISCUSSION AND CONCLUSION}

We developed an approach for extraction of the pathologies of the maxillofacial massif in the scanographiques images. The proposed method is based on the creation of a statistical reference model of the massif allowing, eventually, facilitating the analysis of disharmonies by an automatic comparison with regard to this model.

Despite the advantages of our approach, it presents some limitations: the limited number of image-based and correlation of voxels, because the law of Fisher is based on the fact that the voxels are independent, which may be considered a rough

approximation may affect the results.

Finally, when we see the encouraging results, a contrario approach was very powerful. Especially since she has a major advantage that it is a region-based approach. This made more tracks are possible as the combination of $\mathrm{p}$-values using parametric functions.

\section{REFERENCES}

[1] F. Rousseau, S. Faisan, F. Heitz, J.-P. Armspach, Y. Chevalier, F. Blanc, J. De Seze, and L. Rumbach, "An a contrario approach for change detection in 3D multimodal images: Application to multiple sclerosis in MRI,” IEEE EMBS, 2007.

[2] R.Ramousse R., M. Le Berre M. and L. Le Guelte . "Introduction aux statistiques". 1996. http://www.cons-dev.org/elearning/stat/

[3] F. Mkadmini, S. Faisan, N. Khlifa, F. Rousseau, K. Hamrouni, C. Meyer. Détection de régions pathologiques du massif facial à partir d'images 3D CT par une approche a contrario étendue, 22e Colloque GRETSI 2009.

[4] R.Ben Abdnnour, P.Borne, M.Ksouri and F. M'sahli 'Identification et commande numérique des procédés industriels". Édition technip.2001.

[5] J. Ashburner and K.J. Friston, "Voxel-based morphometry - the methods," NeuroImage, vol. 11, pp. 805-821, 2000.

[6] J. B. Tenenbaum, V. de Silva, and J. C. Langford, "A global geometric framework for nonlinear dimensionality reduction,” Science, vol. 290, no. 5500, pp. 2319-2323, December 2000.

[7] A. Desolneux, L. Moisan, and J.-M. Morel, "A grouping principle and four applications," IEEE Transactions on Pattern Analysis and Machine Intelligence, vol. 25, no. 4, pp. 508-513, April 2003.

[8] T. Veit, F. Cao, and P. Bouthemy, "An a contrario decision framework for region-based motion detection," International Journal on Computer Vision, vol. 68, no. 2, pp. 163-178, 1998. 